\title{
Reappraisal of criticality for two-layer flows and its role in the generation of internal solitary waves
}

\author{
Thomas J. Bridges and Neil M. Donaldson \\ Department of Mathematics, University of Surrey, \\ Guildford, Surrey, GU2 $7 X H$, England
}

May 9, 2007

\begin{abstract}
A geometric view of criticality for two-layer flows is presented. Uniform flows are classified by diagrams in the momentum-massflux space for fixed Bernoulli energy, and cuspoidal curves on these diagrams correspond to critical uniform flows. Restriction of these surfaces to critical flow leads to new sub-surfaces in energy-massflux space. While the connection between criticality and the generation of solitary waves is well known, we find that the nonlinear properties of these bifurcating solitary waves are also determined by the properties of the criticality surfaces. To be specific, the case of two layers with a rigid lid is considered, and application of the theory to other multi-layer flows is sketched.
\end{abstract}

\section{Introduction}

The inviscid flow of two layers of homogeneous fluid of differing density is one of the fundamental simplified models for stratified flow in the atmosphere and ocean. Criticality of uniform flows provides an organizing center for the bifurcation of internal shocks and solitary waves (see recent reviews of GrimshaW [16] and Helfrich \& MELville [17] and references therein). In this paper a new perspective on criticality of uniform flows in the two layer system and its implication is presented. For definiteness, attention will be restricted to the case of a rigid lid, as shown schematically in Figure 1.

Our results are not new in the sense that the condition for criticality for two-layer uniform flows is well understood (e.g. ARMi [1], BAINes [2], BüHLER [10] and references therein), and the existence of solitary waves bifurcating at criticality is also well understood, indeed even to finite amplitude (e.g. Benjamin [4], Choi \& Camassa [11], Dias \& Vandenbroeck [13], Funakoshi \& Oikawa [15], Laget \& Dias [18], Long [19], Pullin \& Grimshaw [20]). However, this paper gives a new perspective on criticality, showing that the geometry of the surfaces of uniform flows, and the surfaces of criticality give basic information about the flow

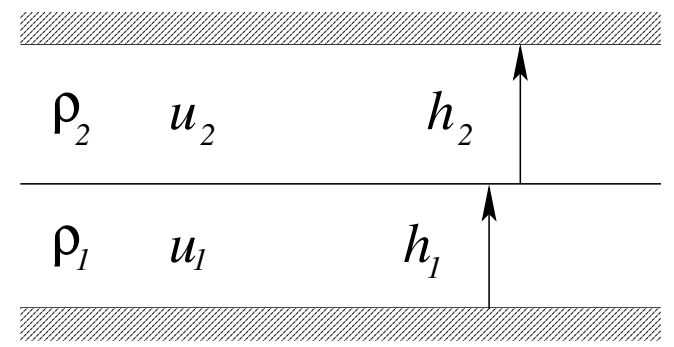

Figure 1: Schematic of the two layer flow with a rigid lid. 


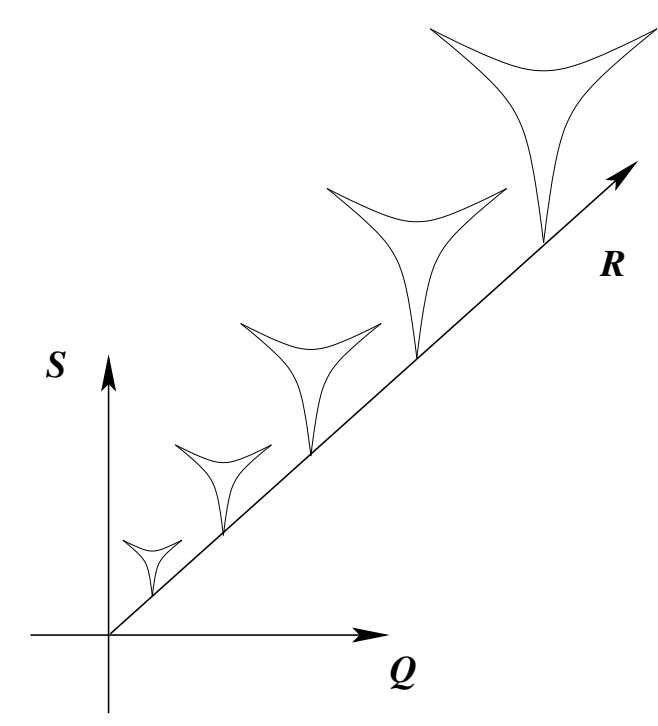

Figure 2: Slices of constant $R$ with $R>0$ of the surface in $(S, Q, R)$ space defined by (1.1).

field, including the prediction of nonlinear properties of bifurcating solitary waves. The geometric view of the uniform flows organizes the flow parameters in a precise way. This latter property is particularly valuable when considering the effect of mean flow on non-trivial internal waves (see [9] for the analogous result for one-layer flows).

To give some idea of the strategy in the paper, the theory for single layer flows is first reviewed. The critical functionals for single-layer uniform flow of unit density, depth $h$ and velocity $u$ are

$$
R=g h+\frac{1}{2} u^{2}, \quad Q=h u, \quad S=h u^{2}+\frac{1}{2} g h^{2},
$$

where $g>0$ is the gravitational constant. These functionals are the Bernoulli energy, mass flux and momentum flux respectively. For given values of $R$ and $Q$, uniform flows correspond to solutions of the first two equations - when the mapping from $(R, Q)$ to $(h, u)$ is uniquely invertible; that is when

$$
\operatorname{det}\left[\begin{array}{ll}
R_{h} & R_{u} \\
Q_{h} & Q_{u}
\end{array}\right] \neq 0
$$

The flow is defined to be critical when the mapping is not uniquely invertible:

$$
0=\operatorname{det}\left[\begin{array}{ll}
R_{h} & R_{u} \\
Q_{h} & Q_{u}
\end{array}\right]=\operatorname{det}\left[\begin{array}{ll}
g & u \\
u & h
\end{array}\right]=g h-u^{2},
$$

which recovers the familiar condition in terms of the Froude number: $1=F^{2}:=\frac{u^{2}}{g h}$. It can be shown that this definition of criticality is equivalent to the degeneracy of the linear long wave speed. See Appendix A of [1] for the definition of criticality in terms of linear long wave speed, and see [9] for the equivalence with the definition used here.

The way to view uniform flows - including the momentum flux - is to plot them in the three-dimensional $(R, Q, S)$ space as shown in Figure 2. This figure shows slices through the surface of uniform flows for fixed $R>0$. For fixed $R>0$ the depth of a uniform flow satisfies

$$
h=\frac{1}{g} R-\frac{1}{2 g} u^{2} .
$$

Substituting this expression into $Q$ and $S$ gives a parameterized curve in the $Q-S$ plane:

$$
\left.\begin{array}{l}
Q(u)=\frac{R}{g} u-\frac{1}{2 g} u^{3} \\
S(u)=\frac{R^{2}}{2 g}+\frac{R}{2 g} u^{2}-\frac{3}{8 g} u^{4}
\end{array}\right\} \quad-\sqrt{2 R}<u<\sqrt{2 R} .
$$




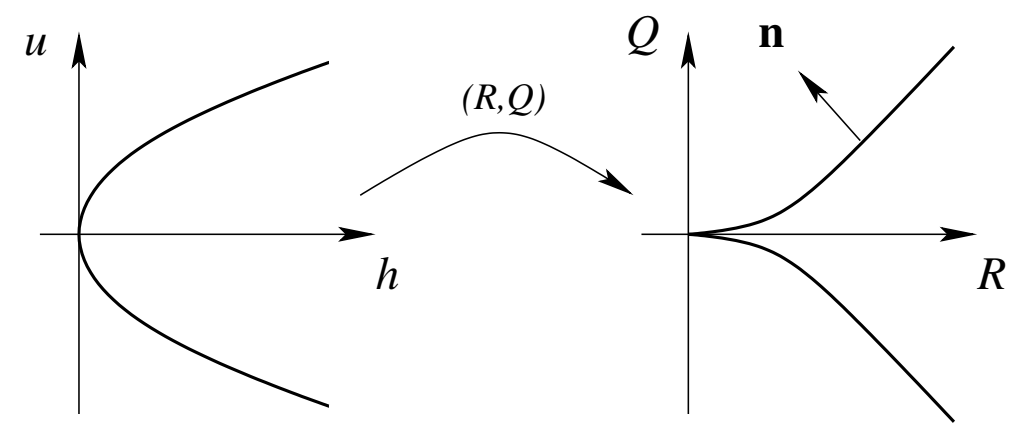

Figure 3: Geometry of the mapping from $(h, u)$ space to $(R, Q)$ space of the criticality curve $g h-u^{2}=0$.

For any fixed $R$, the range of $u$ is bounded: $u^{2}<2 R$. The idea of plotting curves of uniform flows (and more general flows) in the SQR space was first proposed by BENJAMIN \& Lighthill [6]. However, it was Sewell \& Porter [22] (see also page 379 of Sewell [21]) who first recognized the importance of plotting in the full three-dimensional space.

The curves of uniform flow are similar for any $R>0$. The cusp points on the curves with $R$ fixed correspond to critical flows, and are computed from $Q^{\prime}(u)=S^{\prime}(u)=0$ ( $R$ fixed). In the limit as $R \rightarrow 0$ the two curves of cusp points join together in a third cusp at $S=R=Q=0$. The projection onto the $(R, Q)$ - plane of the two curves of criticality is shown on the right in Figure 3.

Critical uniform flows consist of points in the $(h, u)$-plane satisfying $g h=u^{2}$. This curve is shown on the left in Figure 3. The image of this curve in the $(R, Q)$ plane is shown in the right figure.

There is important geometry in these figures which shows up in the analysis of criticality and in the bifurcation of solitary waves. The vector labelled $\mathbf{n}$ in Figure 3 is the normal vector to the curve of criticality in the $(R, Q)$ plane. This vector is also in the kernel of the Jacobian of the mapping $(h, u) \mapsto(R(h, u), Q(h, u))$ :

$$
\operatorname{Jac}(h, u) \mathbf{n}=0, \quad \text { where } \quad \operatorname{Jac}(h, u):=\left[\begin{array}{ll}
R_{h} & R_{u} \\
Q_{h} & Q_{u}
\end{array}\right] .
$$

The vector $\mathbf{n}$ plays another role in determining the location of the cusp in the $(R, Q)$ - plane. The cusp point in the $(R, Q)$ - plane is the image of the point on the curve $g h-u^{2}=0$ where $\mathbf{n}$ is tangent to the curve. Let $f(h, u)=g h-u^{2}$. Then the criticality curve is $f^{-1}(0)$ and $\mathbf{n}$ is tangent to this curve if $\nabla f \cdot \mathbf{n}=0$. Now, $\mathbf{n}=n_{0}(-u, g)$ where $n_{0}$ is a non-zero multiplicative constant, and so

$$
\nabla f \cdot \mathbf{n}=-3 g n_{0} u
$$

Hence $\nabla f \cdot \mathbf{n}=0$ on $f^{-1}(0)$ when $(h, u)=(0,0)$. While it is obvious from the figure that the cusp point is the image of $(h, u)=(0,0)$ this more elaborate geometrical construction will be necessary when considering higher-dimensional surfaces that appear in two-layer flow.

This geometry shows up when considering the solitary waves that bifurcate at criticality. The appropriate model for bifurcating weakly nonlinear solitary waves is the steady KdV equation. Write the $\mathrm{KdV}$ equation for the wave height $\eta$ in the form

$$
\eta_{t}+\kappa \eta \eta_{x}-\tau \eta_{x x x}=0
$$

where $\tau$ is proportional to the Bond number minus $\frac{1}{3}$ (see equation (5.15) for the analogous coefficient for two-layer flow), and $\kappa$ is the coefficient of the nonlinear term. These coefficients 
can be removed by scaling, but there is an interesting connection between $\kappa$ and the geometry of the criticality curve. The steady KdV equation reduces to the ODE

$$
-\tau \eta_{x x}=I-\frac{1}{2} \kappa \eta^{2}
$$

where $I$ is a constant of integration. The coefficient $\kappa$ is determined by the remarkable formula

$$
\kappa=a_{1} \nabla f \cdot \mathbf{n},
$$

where $a_{1}$ is a positive scaling function. See $\S 3$ of [9] for further elaboration of this example. The details of the theory justifying these results can be found in $[8,9,7]$.

The purpose of this paper is to apply these ideas to the case of two-layer flows. There are three features of interest to generalize. First, the set of basic functionals generalizing (1.1) is $R, Q_{1}, Q_{2}$ and $S$, and so the surface of uniform flows is a three-dimensional hypersurface, but with $R$ fixed it is reduced to a two-dimensional surface in $\mathbb{R}^{3}$, generalizing Figure 2 . The cusp curves on this figure correspond to critical flows.

Secondly, the curves shown in Figure 3 will be generalized to a surface associated with a mapping from $\left(h_{1}, u_{1}, u_{2}\right)$ to $\left(R, Q_{1}, Q_{2}\right)$. The condition of criticality for two-layer flows with a rigid lid takes the familiar form

$$
F_{1}^{2}+r F_{2}^{2}=1, \quad F_{j}^{2}=\frac{u_{j}^{2}}{g(1-r) h_{j}^{2}}, \quad r=\frac{\rho_{2}}{\rho_{1}} .
$$

(cf. Armi [1], Baines [2], Bühler [10] and references therein). This condition is normally determined by solving for the normal mode solutions of the time-dependent equations, linearized about uniform flow, and determining conditions for when the characteristic velocities vanish (cf. Appendix A of ARMi [1]). Here it is shown that criticality follows from degeneracy of the Jacobian of the mapping from $\left(h_{1}, u_{1}, u_{2}\right)$ to $\left(R, Q_{1}, Q_{2}\right)$ (see $\S 4$ for details).

The equation (1.3) defines a hypersurface in the three-dimensional $\left(h_{1}, u_{1}, u_{2}\right)$ space, generalizing the left-hand curve in Figure 3. The geometry of this surface, and its image in $\left(R, Q_{1}, Q_{2}\right)$ is studied (see Figure 8 in $\S 4$ ). Thirdly, in $\S 5.1$, it is shown how the geometry of these criticality surfaces predicts the nonlinear properties of the bifurcating internal solitary waves.

\section{Shallow-water two-layer flow with a rigid lid}

The shallow water equations for two layers with a rigid lid take the following form (cf. $§ 3.3$ of Baines [2])

$$
\begin{aligned}
\frac{\partial}{\partial t}\left(\rho_{1} h_{1}\right)+\frac{\partial}{\partial x}\left(\rho_{1} h_{1} u_{1}\right)=0, \\
\frac{\partial}{\partial t}\left(\rho_{2} h_{2}\right)+\frac{\partial}{\partial x}\left(\rho_{2} h_{2} u_{2}\right)=0, \\
\frac{\partial}{\partial t}\left(\rho_{1} u_{1}\right)+\frac{\partial}{\partial x}\left(\frac{1}{2} \rho_{1} u_{1}^{2}+\rho_{1} g h_{1}+\rho_{2} g h_{2}+p_{s}\right)=0, \\
\frac{\partial}{\partial t}\left(\rho_{2} u_{2}\right)+\frac{\partial}{\partial x}\left(\frac{1}{2} \rho_{2} u_{2}^{2}+\rho_{2} g h_{1}+\rho_{2} g h_{2}+p_{s}\right)=0,
\end{aligned}
$$

where $p_{s}$ is the pressure at the rigid lid. Accompanying these equations is the rigid lid constraint $d=h_{1}+h_{2}$, with $d$ a given positive constant. In these equations $u_{1}(x, t)$ and $u_{2}(x, t)$ are the depth-averaged horizontal velocities and $h_{1}(x, t)$ and $h_{2}(x, t)$ are the layer depths. The two Bernoulli equations can be combined by subtracting the fourth equation from the third to eliminate the lid pressure,

$$
\frac{\partial}{\partial t}\left(\rho_{1} u_{1}-\rho_{2} u_{2}\right)+\frac{\partial}{\partial x}\left(\frac{1}{2} \rho_{1} u_{1}^{2}-\frac{1}{2} \rho_{2} u_{2}^{2}+\left(\rho_{1}-\rho_{2}\right) g h_{1}\right)=0 .
$$


Some comments on the role of the lid pressure in the computation of uniform flows is given in Appendix A.

Steady flows - which in this case are uniform flows - satisfy these equations with the timederivative terms dropped. Uniform flows therefore correspond to constant values of $\left(R, Q_{1}, Q_{2}\right)$ with

$$
\begin{aligned}
R & =\frac{1}{2} \rho_{1} u_{1}^{2}-\frac{1}{2} \rho_{2} u_{2}^{2}+\left(\rho_{1}-\rho_{2}\right) g h_{1} \\
Q_{1} & =\rho_{1} h_{1} u_{1} \\
Q_{2} & =\rho_{2}\left(d-h_{1}\right) u_{2} .
\end{aligned}
$$

The Bernoulli energy $R=R_{1}-R_{2}$ is the composite energy and so it can take negative values. Negative $R$ corresponds to higher energy in the upper layer since $R<0$ implies $R_{2}>R_{1}$.

Accompanying the functions $\left(R, Q_{1}, Q_{2}\right)$ is the reduced momentum flux

$$
S=\rho_{1} h_{1} u_{1}^{2}+\rho_{2}\left(\frac{1}{2} d-h_{1}\right) u_{2}^{2}+\frac{1}{2}\left(\rho_{1}-\rho_{2}\right) g h_{1}^{2} .
$$

This function satisfies

$$
\frac{\partial}{\partial t}\left(\rho_{1} u_{1} h_{1}-\rho_{2} u_{2} h_{1}\right)+\frac{\partial}{\partial x}(S)=0 .
$$

The connection between the reduced momentum flux and the physical momentum flux is discussed in Appendix B.

Our first step in the classification of uniform flows is to take $R$ fixed and look at surfaces of uniform flows in the three-dimensional $\left(Q_{1}, Q_{2}, S\right)$ space.

\section{Uniform flows in $\left(Q_{1}, Q_{2}, S\right)$ space}

The functions in (2.3)-(2.4) can be viewed as a mapping from $\mathbb{R}^{3}=\left\{\left(h_{1}, u_{1}, u_{2}\right): 0<h_{1}<d\right\}$ to $\mathbb{R}^{4}=\left\{S, Q_{1}, Q_{2}, R\right\}$. This hypersurface is a generalization of the surface for a single layer fluid shown in Figure 2.

Taking $R$ fixed, $h_{1}$ can be expressed as a function of $r, R$, and the velocities

$$
h_{1}=\frac{2 R-\rho_{1} u_{1}^{2}+\rho_{2} u_{2}^{2}}{2 \rho_{1} g(1-r)} .
$$

Substituting this expression for $h_{1}$ into the equations for $Q_{1}, Q_{2}$ and $S$ with fixed $r$, and $R$ results in a parameterized hypersurface in the three-dimensional $\left(Q_{1}, Q_{2}, S\right)$ space,

$$
\begin{aligned}
\tilde{Q}_{1}\left(\tilde{u}_{1}, \tilde{u}_{2}\right) & =\frac{\tilde{u}_{1}}{2(1-r)}\left(2 \mathscr{R}-\tilde{u}_{1}^{2}+r \tilde{u}_{2}^{2}\right) \\
\tilde{Q}_{2}\left(\tilde{u}_{1}, \tilde{u}_{2}\right) & =\frac{\tilde{u}_{2}}{2(1-r)}\left(2(1-r)-2 \mathscr{R}+\tilde{u}_{1}^{2}-r \tilde{u}_{2}^{2}\right), \\
\tilde{S}\left(\tilde{u}_{1}, \tilde{u}_{2}\right) & =-\frac{1}{8(1-r)}\left(3 \tilde{u}_{1}^{4}-6 r \tilde{u}_{1}^{2} \tilde{u}_{2}^{2}+3 r^{2} \tilde{u}_{2}^{4}-4 \mathscr{R} \tilde{u}_{1}^{2}+4 r(\mathscr{R}+r-1) \tilde{u}_{2}^{2}-4 \mathscr{R}^{2}\right),
\end{aligned}
$$

where the tilde variables are defined by

$$
\begin{gathered}
\tilde{u}_{1}=\frac{u_{1}}{\sqrt{g d}}, \quad \tilde{u}_{2}=\frac{u_{2}}{\sqrt{g d}}, \quad \tilde{h}_{1}=\frac{h_{1}}{d}, \quad \tilde{h}_{2}=\frac{h_{2}}{d}, \\
\tilde{Q}_{1}=\frac{Q_{1}}{\rho_{1} d \sqrt{g d}}, \quad \tilde{Q}_{2}=\frac{Q_{2}}{\rho_{2} d \sqrt{g d}}, \quad \tilde{R}=\frac{R}{\rho_{1} g d}, \quad \tilde{S}=\frac{S}{\rho_{1} g d^{2}},
\end{gathered}
$$

and $\mathscr{R}$ represents the value of $\tilde{R}$. The surface is parameterized by $\tilde{u}_{1}$ and $\tilde{u}_{2}$. The feasible region in velocity space is determined by requiring $h_{1}>0$ and $h_{2}=d-h_{1}>0$. Using (3.5) these constraints lead to

$$
\frac{\tilde{u}_{1}^{2}}{r}-\frac{2 \mathscr{R}}{r}<\tilde{u}_{2}^{2}<\frac{\tilde{u}_{1}^{2}}{r}+\frac{2}{r}(1-r-\mathscr{R}) .
$$




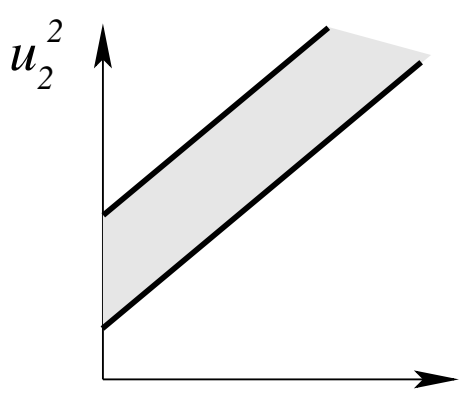

Case 1

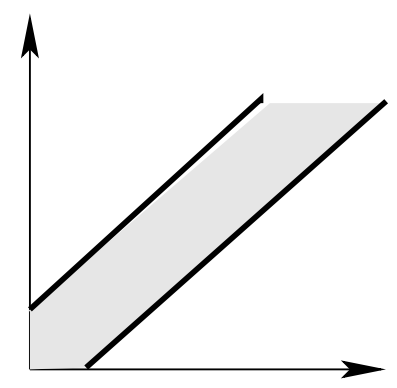

Case 2

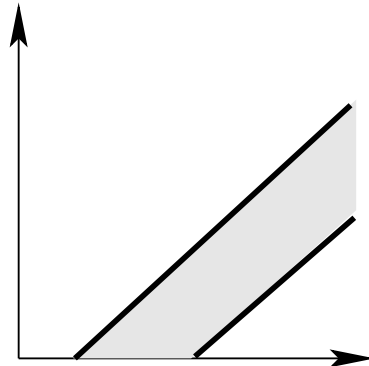

Case 3 $u_{1}^{2}$

Figure 4: Schematic of feasible regions of uniform flows in velocity space. The shaded regions (which extend to infinity) consist of admissible velocity fields for uniform flow. Equations for the upper and lower boundaries are given in equation (3.7). Case 1: $\mathscr{R}<0$, Case 2: $0<\mathscr{R}<1-r$, Case 3: $\mathscr{R}>1-r$.

In the velocity space $\left(\tilde{u}_{1}^{2}, \tilde{u}_{2}^{2}\right)$ these constraints define an infinite strip bounded above and below by parallel straight lines with slope $r^{-1}$. There are three cases depending on whether the energy is predominantly in the upper or lower layer. A schematic of the three cases is shown in Figure 4. Case 1 corresponds to $\mathscr{R}<0$. In this case the upper fluid energy is dominant since $R_{2}>R_{1}$. Case 2 corresponds to $0<\mathscr{R}<1-r$, where the energy in the upper and lower layers is in balance. Case 3 corresponds to $\mathscr{R}>1-r$, and in this case the lower fluid energy is dominant.

To plot surfaces of uniform flows in the scaled $\left(Q_{1}, Q_{2}, S\right)$ space an upper limit is chosen for $\tilde{u}_{1}^{2}$ which ensures that the interesting features of the surfaces (the criticality regions) are included. The three sets of surfaces, corresponding to examples for Cases 1, 2, and 3 respectively, are shown in Figures 5, 6 and 7. The parameter values for each figure are

- Figure 5 (Case 1): $r=0.1, \mathscr{R}=-0.1$ and $\left(\tilde{u}_{1}^{2}\right)_{\max }=0.5$

- Figure 6 (Case 2): $r=0.1, \mathscr{R}=0.5$ and $\left(\left(\tilde{u}_{1}^{2}\right)_{\max }=1.5\right.$

- Figure 7 (Case 3): $r=0.1, \mathscr{R}=1.0$ and $\left(\left(\tilde{u}_{1}^{2}\right)_{\max }=2.5\right.$.

Surprisingly, the qualitative aspects of the criticality surfaces are independent of the choice of $r$ and the Case. This is shown in equation (4.8) and discussed in $\S 4$.

To obtain the surfaces in figures 5(c), 6(c) and 7(c) showing the criticality regions in closer detail we have set the maximum value of $\tilde{u}_{1}^{2}$ to be $0.1,0.6$ and 0.8 respectively. Also in figures $5(\mathrm{c})$ and $6(\mathrm{c})$ the maximum value of $\tilde{u}_{2}^{2}$ is set to be 10 and 5 respectively. In these figures the curves of critical uniform flows are colored in red. These figures are best viewed interactively in MAPLE where they can be rotated and enlarged, and copies of the MAPLE codes are available for downloading ${ }^{1}$.

\subsection{Criticality parabolae in velocity space}

In part (b) of Figures 5, 6, and 7 the curve of criticality with $r, \mathscr{R}$ fixed, in velocity space is shown. This curve is computed as follows. Take the well known condition for critical flow (1.3) in terms of scaled variables,

$$
\tilde{F}_{1}^{2}+r \tilde{F}_{2}^{2}=1, \quad \text { with } \quad \tilde{F}_{1}^{2}=\frac{\tilde{u}_{1}^{2}}{(1-r) \tilde{h}_{1}} \quad \text { and } \quad \tilde{F}_{2}^{2}=\frac{\tilde{u}_{2}^{2}}{(1-r)\left(1-\tilde{h}_{1}\right)} .
$$

\footnotetext{
${ }^{1}$ http://www.maths. surrey.ac.uk/personal/st/T.Bridges/RIGID-LID/
} 


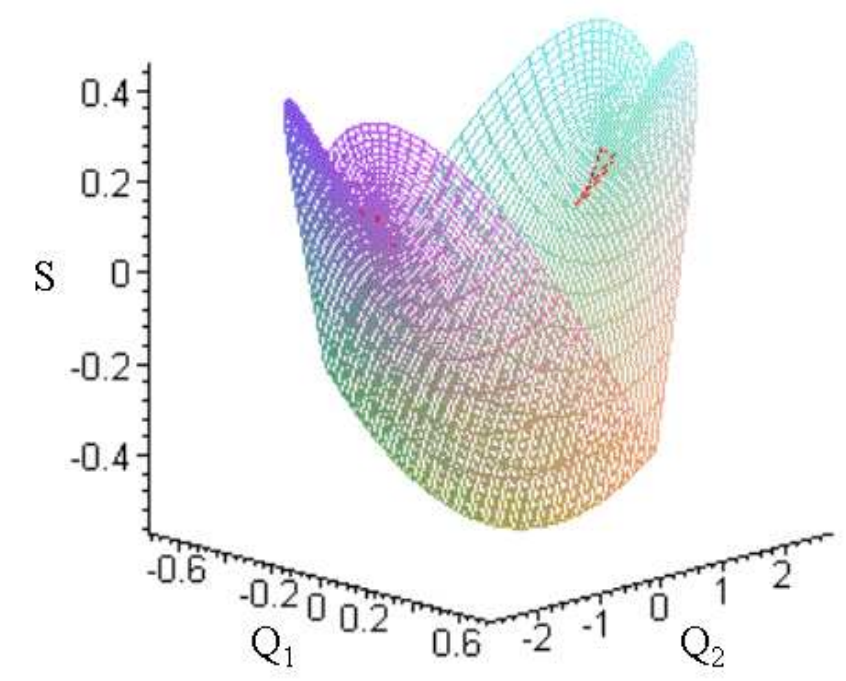

(a) The surface of uniform flows associated with Case 1, with $\left(\tilde{u}_{1}^{2}\right)_{\max }=0.5$.
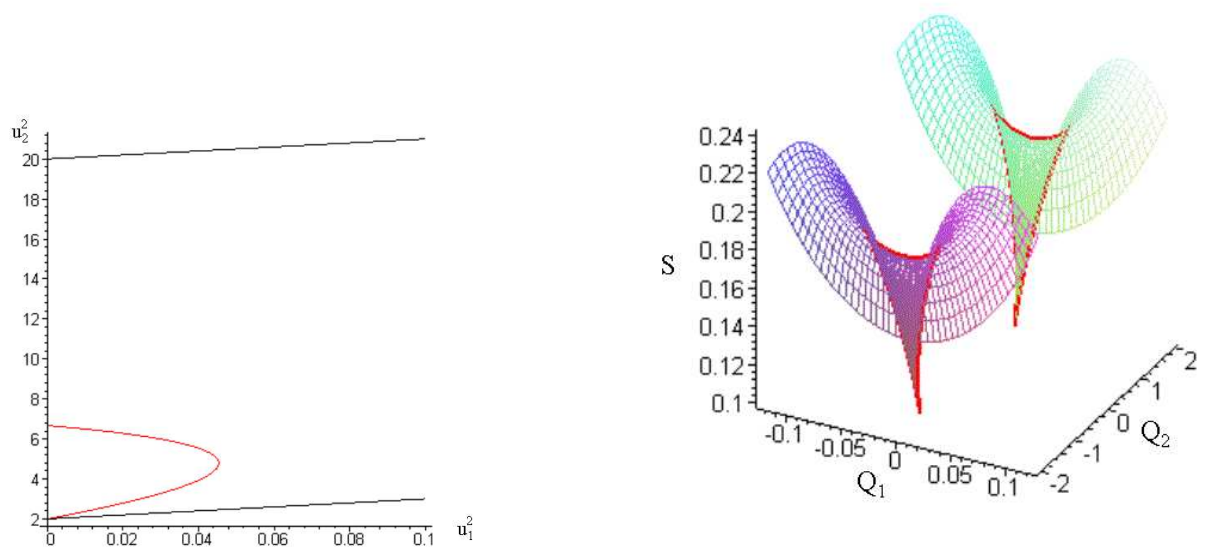

(b) The criticality parabola in velocity (c) Detailed view of the surface in (a) with the criticality curve space. highlighted.

Figure 5: Surface of uniform flows and criticality curves corresponding to Case 1, in terms of scaled variables with $r=0.1$ and $\mathscr{R}=-0.1$. 


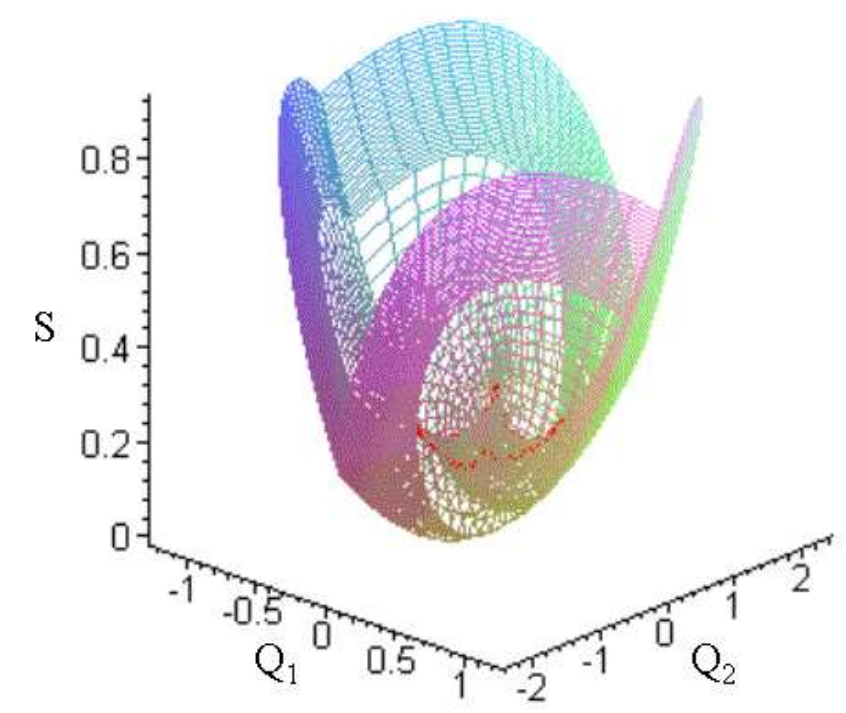

(a) The surface of uniform flows associated with Case 2, with $\left(\tilde{u}_{1}^{2}\right)_{\max }=1.5$.
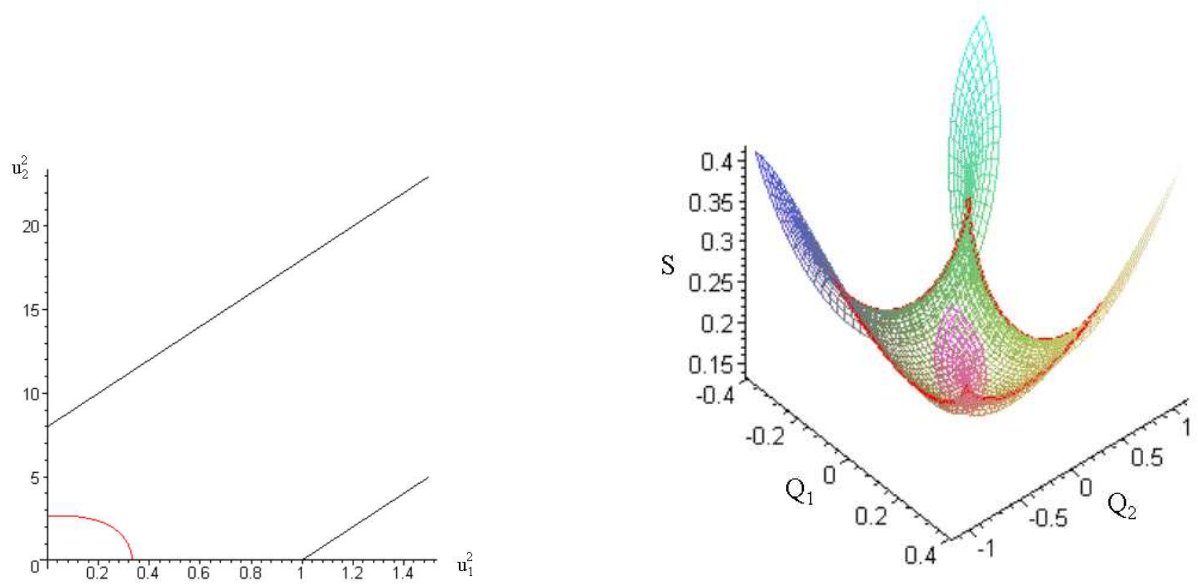

(b) The criticality parabola in velocity (c) Detailed view of the surface in (a) with the criticality curve space. highlighted.

Figure 6: Surface of uniform flows and criticality curves corresponding to Case 2, in terms of scaled variables with $r=0.1$ and $\mathscr{R}=0.5$. 


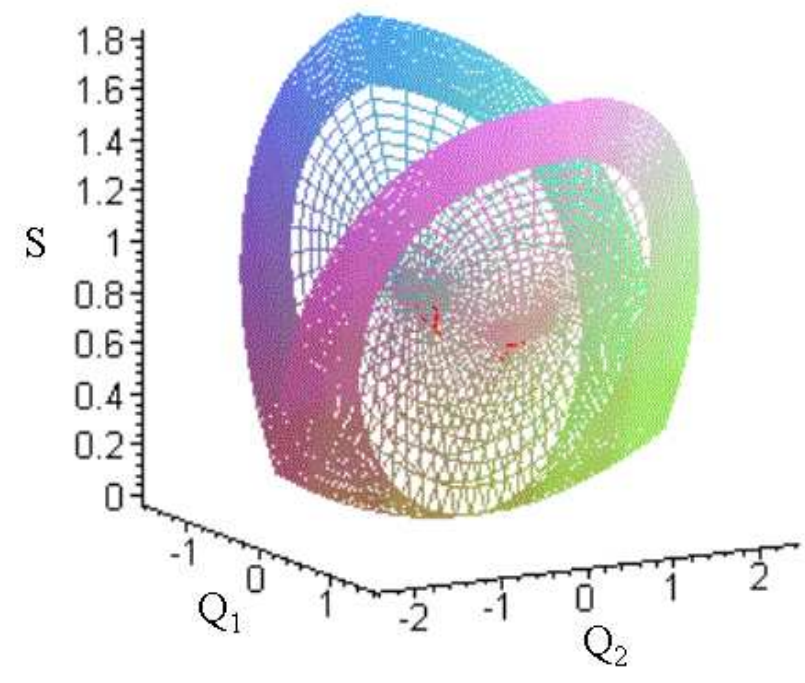

(a) The surface of uniform flows associated with Case 2, with $\left(\tilde{u}_{1}^{2}\right)_{\max }=2.5$.
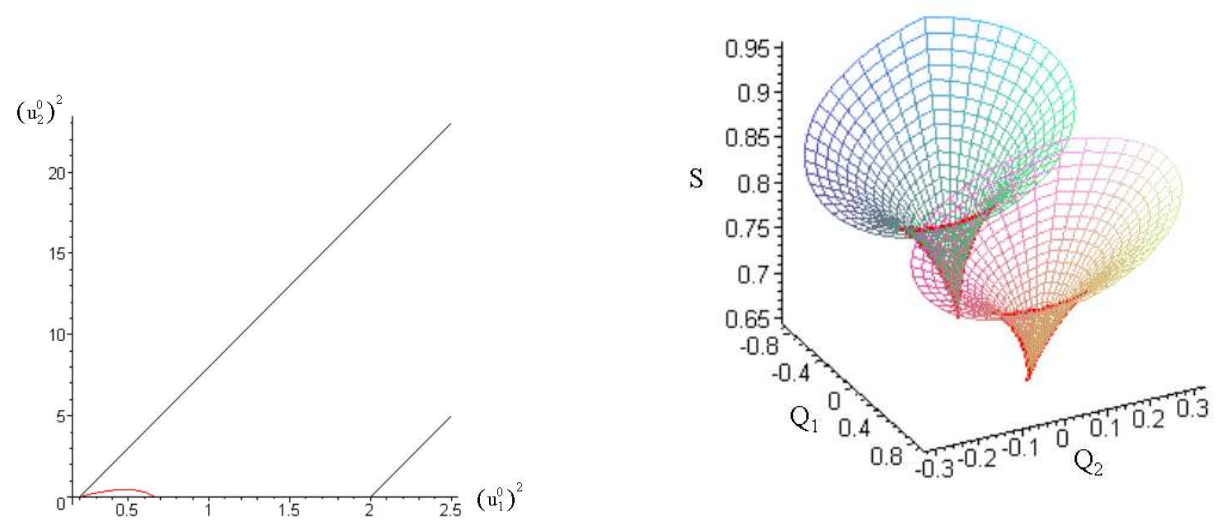

(b) The criticality parabola in velocity (c) Detailed view of the surface in (a) with the criticality curve space. highlighted.

Figure 7: Surface of uniform flows and criticality curves corresponding to Case 3, in terms of scaled variables with $r=0.1$ and $\mathscr{R}=1$. 
Eliminate $\tilde{h}_{1}$ in this equation using (3.5) and let $\tilde{u}_{1}^{2}=U$ and $\tilde{u}_{2}^{2}=V$; then the curve of criticality in velocity space is a quadratic in $(U, V)$,

$$
3 U^{2}-6 r U V+3 r^{2} V^{2}+(6(1-r)-8 \mathscr{R}) U+(8 r \mathscr{R}-2 r(1-r)) V+4\left(\mathscr{R}^{2}-(1-r) \mathscr{R}\right)=0 .
$$

This equation is a parabola in $U$ and $V$ since the discriminant, $(-3 r)^{2}-3\left(3 r^{2}\right)$, is zero, and it can be parameterized by

$U(s)=U_{0}-\alpha s(r s-2), \quad V(s)=V_{0}-\alpha s(2 r+s), \quad \alpha=\frac{r(1-r)}{3\left(1+r^{2}\right)^{2}}, \quad$ with $\quad-\infty<s<\infty$,

where $U_{0}$ and $V_{0}$ are the coordinates of the vertex of the parabola,

$$
U_{0}=\frac{\left(-r^{3}+2 \mathscr{R} r^{2}+r^{2}+r+2 \mathscr{R}-1\right)\left(-r^{3}+2 \mathscr{R} r^{2}+r^{2}-3 r+2 \mathscr{R}+3\right)}{12(1-r)\left(1+r^{2}\right)^{2}},
$$

and

$$
V_{0}=\frac{1}{r} U_{0}-\frac{4 \mathscr{R}}{3 r}+\frac{(1-r)\left(3+r^{2}\right)}{3 r\left(1+r^{2}\right)} .
$$

The intersection of this curve with the region of uniform flows gives the critical uniform flows. In part (b) of Figures 5, 6, and 7 the criticality parabolae are colored in red.

\section{Geometry of the criticality surfaces in $\left(R, Q_{1}, Q_{2}\right)$ space}

The surfaces in $\S 3$ show all uniform flows, with the critical uniform flows showing up as singular curves on the surfaces. However, in the four dimensional space $\left(S, R, Q_{1}, Q_{2}\right)$ the critical uniform flows form a $2 \mathrm{D}$ surface. In this section, the $2 \mathrm{D}$ critical surfaces are projected into $\mathbb{R}^{3}$. It is these surfaces that contain the geometric information about the bifurcation of internal solitary waves.

Uniform flows are relative equilibria, and they are critical precisely when the relative equilibrium characterization is degenerate. The details of this analysis for two-layer flows is given in $\S 4.3$ of Donaldson [14]. The upshot is that uniform flows are critical precisely when the mapping

$$
\left(h_{1}, u_{1}, u_{2}\right) \mapsto\left(R\left(h_{1}, u_{1}, u_{2}\right), Q_{1}\left(h_{1}, u_{1}, u_{2}\right), Q_{2}\left(h_{1}, u_{1}, u_{2}\right)\right),
$$

given in (2.3) is degenerate,

$$
\operatorname{det}\left[\frac{\partial\left(R, Q_{1}, Q_{2}\right)}{\partial\left(h_{1}, u_{1}, u_{2}\right)}\right]=0 .
$$

First note that $\left(R, Q_{1}, Q_{2}\right)$ is a gradient mapping. Let

$$
\mathscr{F}(\mathbf{c})=\frac{1}{2} \rho_{1} h_{1} u_{1}^{2}+\frac{1}{2} \rho_{2}\left(d-h_{1}\right) u_{2}^{2}+\frac{1}{2}\left(\rho_{1}-\rho_{2}\right) g h_{1}^{2} .
$$

Then

$$
\begin{aligned}
R & :=\frac{\partial \mathscr{F}}{\partial h_{1}}=\frac{1}{2} \rho_{1} u_{1}^{2}-\frac{1}{2} \rho_{2} u_{2}^{2}+\left(\rho_{1}-\rho_{2}\right) g h_{1} \\
Q_{1} & :=\frac{\partial \mathscr{F}}{\partial u_{1}}=\rho_{1} h_{1} u_{1} \\
Q_{2} & :=\frac{\partial \mathscr{F}}{\partial u_{2}}=\rho_{2}\left(d-h_{1}\right) u_{2} .
\end{aligned}
$$

It follows from the gradient structure that the Jacobian in (4.2) is symmetric.

To simplify notation, let

$$
\mathbf{c}:=\left(h_{1}, u_{1}, u_{2}\right) \quad \text { and } \quad \mathbf{P}(\mathbf{c}):=\left(R(\mathbf{c}), Q_{1}(\mathbf{c}), Q_{2}(\mathbf{c})\right) .
$$

Then the Jacobian $\mathrm{DP}(\mathbf{c})$ is

$$
\mathrm{DP}(\mathbf{c})=\left[\begin{array}{ccc}
\left(\rho_{1}-\rho_{2}\right) g & \rho_{1} u_{1} & -\rho_{2} u_{2} \\
\rho_{1} u_{1} & \rho_{1} h_{1} & 0 \\
-\rho_{2} u_{2} & 0 & \rho_{2}\left(d-h_{1}\right)
\end{array}\right],
$$


and so

$$
\operatorname{det}(\mathrm{DP}(\mathbf{c}))=\rho_{1} \rho_{2}\left(\rho_{1}-\rho_{2}\right) g h_{1}\left(d-h_{1}\right)\left[1-F_{1}^{2}-r F_{2}^{2}\right],
$$

where $F_{1}^{2}$ and $F_{2}^{2}$ are as defined in (1.3). Setting $\operatorname{det}(\mathrm{DP}(\mathbf{c}))=0$ recovers the familiar condition for criticality of two-layer uniform flows with a rigid lid.

At criticality, the Jacobian has rank 2 (it is not further deficient). This statement is confirmed by noting that $\mathrm{DP}(\mathbf{c})$ has rank two if

$$
\Delta^{\prime}(0) \neq 0 \quad \text { where } \quad \Delta(\lambda)=\operatorname{det}[\mathrm{DP}(\mathbf{c})-\lambda \mathbf{I}], \quad \text { when } \quad \Delta(0)=\operatorname{det}(\mathrm{DP}(\mathbf{c}))=0 .
$$

Expanding out the determinant and differentiating

$$
\Delta^{\prime}(0)=\rho_{1}^{2}\left[u_{1}^{2}+r^{2} u_{2}^{2}-r h_{1}\left(d-h_{1}\right)-(1-r) g h_{1}-r(1-r) g\left(d-h_{1}\right)\right] .
$$

But criticality implies

$$
\frac{u_{1}^{2}}{g h_{1}}+r \frac{u_{2}^{2}}{g\left(d-h_{1}\right)}=(1-r) .
$$

Substituting this expression into $\Delta^{\prime}(0)$ results in

$$
\Delta^{\prime}(0)=-\frac{r \rho_{1}^{2}}{h_{1}\left(d-h_{1}\right)}\left[h_{1}^{2} u_{1}^{2}+\left(d-h_{1}\right)^{2} u_{2}^{2}+h_{1}^{2}\left(d-h_{1}\right)^{2}\right],
$$

which is strictly negative when $0<h_{1}<d$, confirming that the rank of $\mathrm{DP}(\mathbf{c})$ is two.

The condition $\operatorname{det}[\mathrm{DP}(\mathbf{c})]=0$ defines a hypersurface in $\mathbf{c}$ space, and this surface can be parameterized by taking

$$
h_{1}(s, \varphi)=s d, \quad u_{1}(s, \varphi)=\sqrt{g(1-r) s d} \cos \varphi, \quad u_{2}(s, \varphi)=\sqrt{\frac{g d(1-r)(1-s)}{r}} \sin \varphi .
$$

For fixed $r$ this parameterization maps out a surface in $\left(h_{1}, u_{1}, u_{2}\right)$ space by taking $0 \leq s \leq 1$ and $0 \leq \varphi<2 \pi$. An example, taking $r=0.1$ is shown in Figure 8. The image of the criticality surface in $\left(R, Q_{1}, Q_{2}\right)$ space is also shown in Figure 8. The second surface is obtained by substituting (4.7) into (4.3). These figures generalize those for one layer shown in Figure 3.

The surface in $\left(h_{1}, u_{1}, u_{2}\right)$ space has an elliptic cross-section, with rotating axes as $h_{1}$ increases. When $h_{1}=0$ it is a straight line (degenerate ellipse) in the $u_{2}$ direction and when $h_{1}=1$ it is a straight line (again a degenerate ellipse) in the $u_{1}$ direction. Since $r=0.1$ is not a typical value for open ocean applications, it is useful to note that the surfaces are qualitatively the same for all $r \in(0,1)$. To see this, recast $(4.7)$ as

$$
\frac{h_{1}}{d}=s, \quad \frac{u_{1}}{\sqrt{g(1-r) d}}=\sqrt{s} \cos \varphi, \quad \frac{\sqrt{r} u_{2}}{\sqrt{g(1-r) d}}=\sqrt{(1-s)} \sin \varphi .
$$

The only effect of $r$ is to scale the velocities and to rotate the major and minor axes of the elliptic cross section.

Some remarkable new features show up in the image surface in $\left(R, Q_{1}, Q_{2}\right)$ space. There is a series of cuspoidal curves. These cuspoidal curves can be predicted using singularity theory $[3,7]$. The cusp curves are the image in $\left(R, Q_{1}, Q_{2}\right)$ space of curves where the kernel of $\mathrm{DP}(\mathbf{c})$ is in the tangent space of the criticality surface.

The Jacobian $\mathrm{DP}(\mathbf{c})$ has rank two so the kernel of $\mathrm{DP}(\mathbf{c})$ is one dimensional. A simple calculation shows that

$$
\operatorname{DP}(\mathbf{c}) \mathbf{n}=0 \Rightarrow \mathbf{n}=\mathbb{R}\left(\begin{array}{c}
1 \\
-\frac{u_{1}}{h_{1}} \\
\frac{u_{2}}{\left(d-h_{1}\right)}
\end{array}\right)
$$

where $\mathbb{R}$ represents an arbitrary multiplicative constant. Since it does not affect the later discussion, take the multiplicative constant to be unity. The symbol $\mathbf{n}$ is used since $\mathbf{n}$ can be 

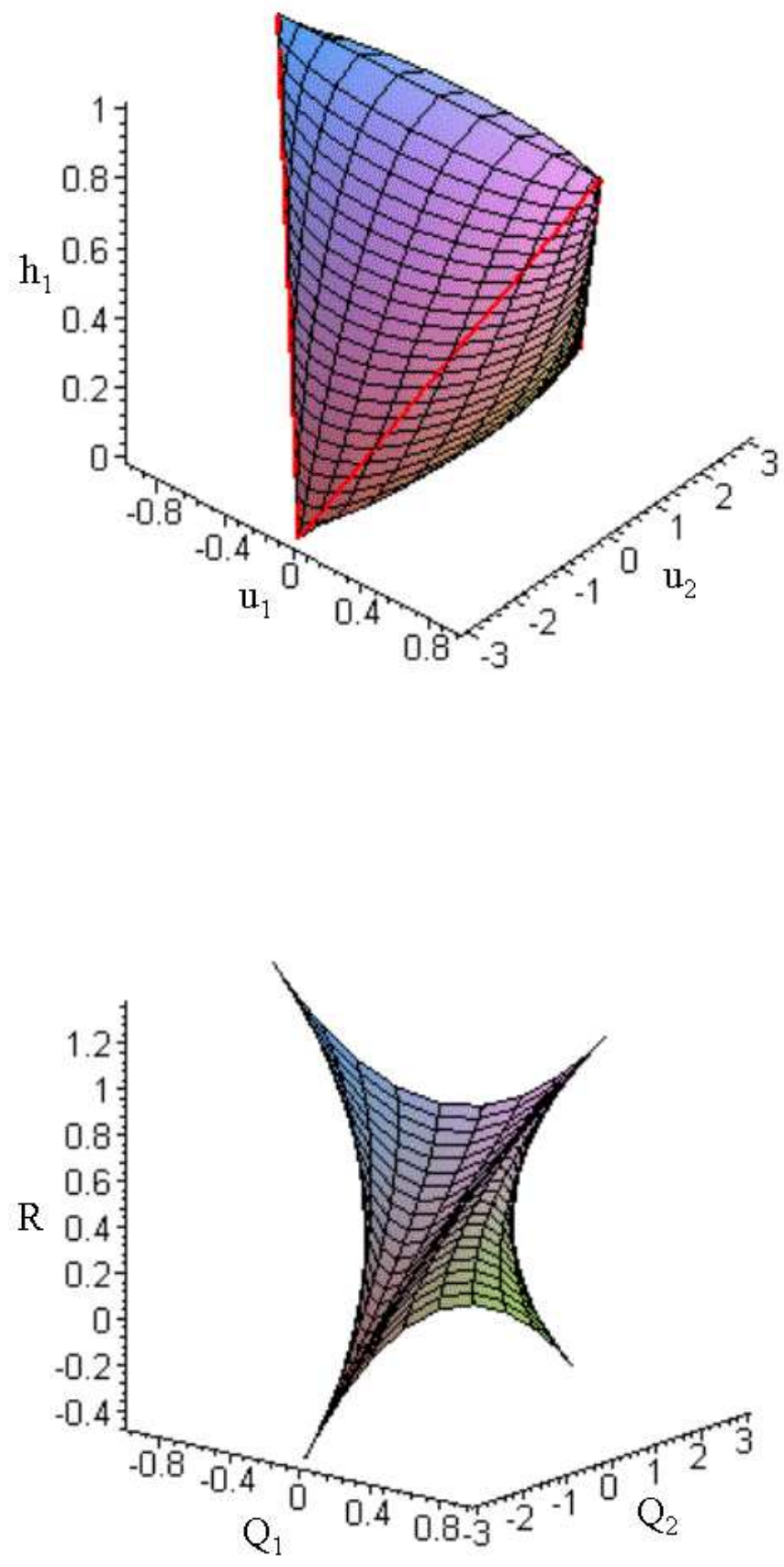

Figure 8: Criticality surfaces when $r=0.1$, with units chosen so that $\rho_{1}=1, g=1$ and $d=1$. 


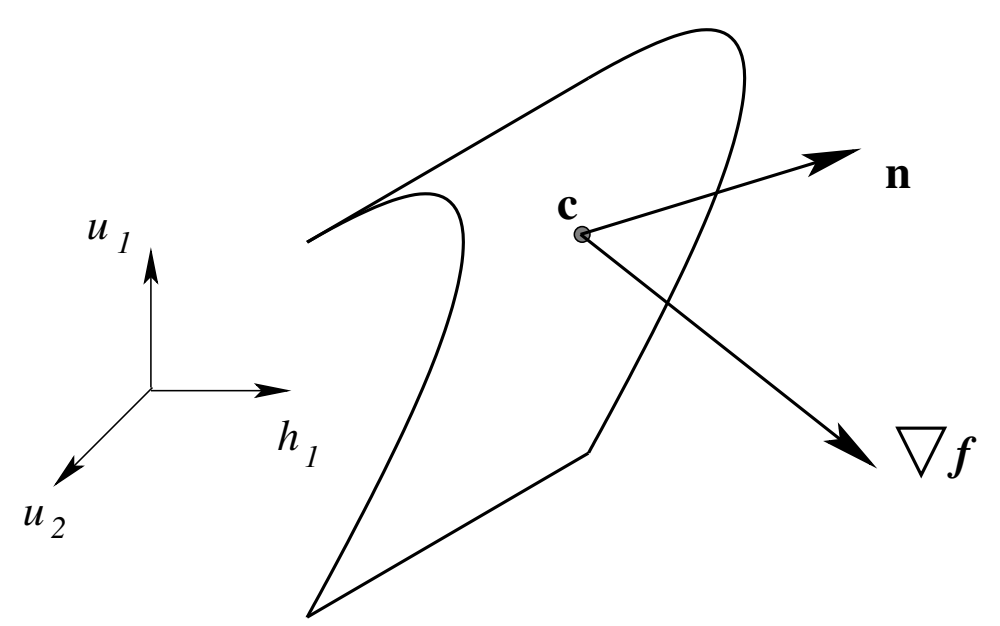

Figure 9: Schematic of the vectors $\nabla f$, where $f(\mathbf{c})=\operatorname{det}(\operatorname{DP}(\mathbf{c}))$, and $\mathbf{n} \in \operatorname{Ker}(\mathrm{DP}(\mathbf{c}))$ at a point $\mathbf{c}=\left(h_{1}, u_{1}, u_{2}\right)$ on the surface of criticality in $\left(h_{1}, u_{1}, u_{2}\right)$ space.

interpreted as a normal vector - to the surface in $\left(R, Q_{1}, Q_{2}\right)$ space - away from the singular curves.

Now define

$$
f(\mathbf{c}):=\operatorname{det}[\mathrm{DP}(\mathbf{c})]=\mathrm{C}\left[(1-r)-\frac{u_{1}^{2}}{g h_{1}}-r \frac{u_{2}^{2}}{g h_{2}}\right], \quad \mathrm{C}=\rho_{1}^{2} \rho_{2} g h_{1} h_{2} .
$$

Then the criticality surface in $\left(h_{1}, u_{1}, u_{2}\right)$ space is defined by $f^{-1}(0)$ and the vector $\mathbf{n}$ is tangent to this surface if $\nabla f \cdot \mathbf{n}=0$. Now,

$$
\nabla f=\frac{\mathrm{C}}{g}\left(\frac{u_{1}^{2}}{h_{1}^{2}}-\frac{r u_{2}^{2}}{h_{2}^{2}},-\frac{2 u_{1}}{h_{1}},-\frac{2 r u_{2}}{h_{2}}\right),
$$

and so

$$
\nabla f \cdot \mathbf{n}=\frac{3 \mathrm{C}}{\rho_{1} g}\left(\rho_{1} \frac{u_{1}^{2}}{h_{1}^{2}}-\rho_{2} \frac{u_{2}^{2}}{h_{2}^{2}}\right) .
$$

The singularity $\rho_{1} u_{1}^{2} / h_{1}^{2}=\rho_{2} u_{2}^{2} / h_{2}^{2}$ is familiar in the theory of solitary waves and will arise in the analysis in the next section. Here it arises purely as a singularity in the criticality surfaces. The more familiar version of this singularity in the literature is the special case $u_{1}^{2}=u_{2}^{2}$ which reduces it to

$$
\frac{h_{2}}{h_{1}}=\sqrt{r}
$$

However the case $u_{1}^{2}=u_{2}^{2}$ is a single curve in the criticality surface in Figure 8 (see $\S 4.1$ for an explicit parameterization of this curve), and the singularity (4.11) then corresponds to a discrete set of points on the criticality surface.

The condition $\nabla f \cdot \mathbf{n}=0$ defines curves on the criticality surface in $\left(h_{1}, u_{1}, u_{2}\right)$ space in Figure 8 . With the parameterization (4.7), the function $\nabla f \cdot \mathbf{n}$ can be put in an illuminating form

$$
\nabla f \cdot \mathbf{n}=\frac{3 \mathrm{C}(1-r)}{h_{1} h_{2}}\left(\left(d-h_{1}\right) \cos ^{2} \varphi-h_{1} \sin ^{2} \varphi\right)
$$

It is immediate that

$$
\nabla f \cdot \mathbf{n}=0 \quad \Rightarrow \quad h_{1}=d \cos ^{2} \varphi .
$$

The origin of $\varphi$ can be chosen so that $\varphi=0$ corresponds to $u_{2}=0$ and $u_{1}>0$. For each fixed $h_{1} \in(0, d)$, the singularity curve $h_{1}=d \cos ^{2} \varphi$ divides the criticality surface into four regions 
as $\varphi$ goes from 0 to $2 \pi$. In two of these regions $\nabla f \cdot \mathbf{n}<0$ and in the other two it is positive. The curve $h_{1}=d \cos ^{2} \varphi$ is highlighted on the criticality surface in Figure 8 .

Yet another characterization of the function $\nabla f \cdot \mathbf{n}$ is in terms of the second derivative of the mapping $\mathbf{P}(\mathbf{c})$ in the direction of $\mathbf{n}$. Let

$$
K(\mathbf{c})=n_{1} R(\mathbf{c})+n_{2} Q_{1}(\mathbf{c})+n_{3} Q_{2}(\mathbf{c}),
$$

where $\mathbf{n}=\left(n_{1}, n_{2}, n_{3}\right)$ is in the kernel of $\mathbf{D P}(\mathbf{c})$. Fix $\mathbf{c}$ to be any point on the criticality surface as in Figure 9 such that $\nabla f \cdot \mathbf{n} \neq 0$. Look at the image in $\left(R, Q_{1}, Q_{2}\right)$ space of the path $\mathbf{c}+s \mathbf{n}$ with $s$ small. The image is the curve $K(\mathbf{c}+s \mathbf{n})$ which has a vanishing first derivative (since $\mathrm{DP}(\mathbf{c}) \mathbf{n}=0)$ and second derivative

$$
\left.\frac{d^{2}}{d s^{2}} K(\mathbf{c}+s \mathbf{n})\right|_{s=0}=3\left(\rho_{1} \frac{u_{1}^{2}}{h_{1}^{2}}-\rho_{2} \frac{u_{2}^{2}}{h_{2}^{2}}\right)=\frac{\rho_{1} g}{\mathrm{C}} \nabla f \cdot \mathbf{n} .
$$

A schematic of $K(s)$ versus $s$ for $s$ small is shown in Figure 10. Note that the image of $\mathbf{c}+s \mathbf{n}$ in $\left(R, Q_{1}, Q_{2}\right)$ space creates a barrier. In other words uniform flows exist on only one side of the criticality surface in $\left(R, Q_{1}, Q_{2}\right)$ space, and which side is determined by the sign of $\nabla f \cdot \mathbf{n}$. On the side of the surface where uniform flows exist, there are two and they are the conjugate flows.

\subsection{The Kelvin-Helmholtz instability}

When $u_{1} \neq u_{2}$ there is the potential for Kelvin-Helmholtz (KH) instability [5]. Without surface tension, all flows with nonzero velocity difference are unstable, and with surface tension there is a critical value of the velocity difference below which the uniform flow is stable. The role of KH instability will not be considered in this paper, as it requires bringing in time dependence. However, it is of interest to look at the velocity difference on the surface of criticality. Using the parameterization (4.7) the velocity difference on the surface of criticality is

$$
\frac{u_{1}^{2}-u_{2}^{2}}{g d(1-r)}=\frac{h_{1}}{d} \cos ^{2} \varphi-\frac{1}{r}\left(1-\frac{h_{1}}{d}\right) \sin ^{2} \varphi .
$$

Hence the velocity difference varies over the criticality surface, and there is a distinguished curve on the criticality surface where the velocity difference vanishes:

$$
\frac{h_{1}}{d}=\frac{\sin ^{2} \varphi}{\sin ^{2} \varphi+r \cos ^{2} \varphi} .
$$

Note that this curve does not intersect the singular curve (4.12). The main observation is that there are lines on the criticality surface where the $\mathrm{KH}$ instability is irrelevant. However, on the criticality surface, away from the curve (4.13), the KH instability will affect the dynamics always when surface tension is zero. When surface tension is non-zero there will be a region around the curve (4.13) where the uniform flow in $\mathrm{KH}$ stable, and $\mathrm{KH}$ unstable outside that region.

\section{The role of criticality in the generation of solitary waves}

To look at the bifurcation of solitary waves, dispersion terms need to be added to the shallow water equations. There are a number of derivations of Boussinesq models for internal waves at the interface between two fluids with a rigid lid (e.g. Chor \& Camassa [11], Craig, Guyenne \& KALISCH [12] and references therein). However, there is an equivalence class of Boussinesq models and we have two important requirements: the Boussinesq model should be Galilean invariant, and it should contain evolution equations for the mass density in the upper and lower layers. The former requirement ensures a duality between a moving frame and a stationary 


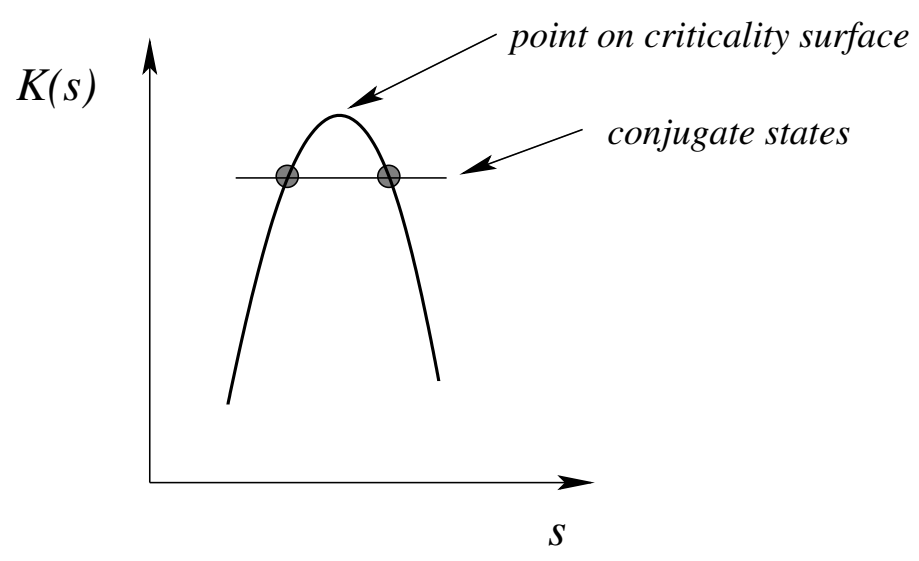

Figure 10: A schematic of the image of the path $x \mapsto \mathbf{c}+s \mathbf{n}$ in the direction $\mathbf{n}$ in $\left(R, Q_{1}, Q_{2}\right)$ space. When $\nabla f \cdot \mathbf{n}$ has the opposite sign, the parabola has a minimum instead of a maximum.

frame with steady velocity. The independent mass equations are needed to ensure independent mean flow in the upper and lower layers. A Boussinesq model satisfying these requirements is derived in $\S 2.5$ of DonaLDSON [14],

$$
\begin{aligned}
\frac{\partial}{\partial t}\left(\rho_{1} h_{1}\right)+\frac{\partial}{\partial x}\left(\rho_{1} h_{1} u_{1}\right) & =0 \\
\frac{\partial}{\partial t}\left(\rho_{2} h_{2}\right)+\frac{\partial}{\partial x}\left(\rho_{2} h_{2} u_{2}\right) & =0 \\
\frac{\partial}{\partial t}\left(\rho_{1} u_{1}-\rho_{2} u_{2}\right)+\frac{\partial}{\partial x}\left(\frac{1}{2} \rho_{1} u_{1}^{2}-\frac{1}{2} \rho_{2} u_{2}^{2}+\left(\rho_{1}-\rho_{2}\right) g h_{1}\right) & =\tau \frac{\partial^{3} h_{1}}{\partial x^{3}},
\end{aligned}
$$

with the constraint $h_{1}+h_{2}=d$. The coefficient of the dispersion term is

$$
\tau=T-\frac{1}{3} \overline{h_{1} h_{2}} g\left(\rho_{1}-\rho_{2}\right) \frac{\left(\rho_{1} \overline{h_{1}}+\rho_{2} \overline{h_{2}}\right)}{\left(\rho_{1} \overline{h_{2}}+\rho_{2} \overline{h_{1}}\right)},
$$

where $T$ is the coefficient of surface tension at the interface, and $\overline{h_{1}}, \overline{h_{2}}$ are reference depths (they can be taken to be equal to the uniform flow). The evolution equation is ill-posed when $\tau<0$ (weak surface tension), but this will not be an issue in this paper as attention will be restricted to the steady part of the equations.

A Hamiltonian formulation of the steady equations is constructed as follows. Introduce new coordinates

$$
Z:=\left(\gamma, \phi_{1}, \phi_{2}, h_{1}, \chi, q_{1}, q_{2}, H\right)
$$

with $\gamma, \phi_{1}$ and $\phi_{2}$ potential functions defined by

$$
\begin{aligned}
& h_{1}=\frac{d \gamma}{d x}, \quad u_{1}=\frac{d \phi_{1}}{d x}, \quad u_{2}=\frac{d \phi_{2}}{d x} \\
& q_{1}=\rho_{1} h_{1} \frac{d \phi_{1}}{d x}, \quad q_{2}=\rho_{2}\left(d-h_{1}\right) \frac{d \phi_{2}}{d x}, \quad H=\tau \frac{d h_{1}}{d x},
\end{aligned}
$$

and $\chi$ is the value of the Bernoulli function, and satisfies $\chi_{x}=0$ (see (5.19) below for elaboration). Then the steady equations are equivalent to the Hamiltonian system

$$
\mathbf{J} Z_{x}=\nabla S(Z), \quad Z \in \mathbb{R}^{8}
$$

with Hamiltonian function

$$
S(Z)=\chi h_{1}-\frac{1}{2}\left(\rho_{1}-\rho_{2}\right) g h_{1}^{2}+\frac{1}{2} \frac{q_{1}^{2}}{\rho_{1} h_{1}}+\frac{1}{2} \frac{q_{2}^{2}}{\rho_{2}\left(d-h_{1}\right)}+\frac{1}{2 \tau} H^{2},
$$


and symplectic operator

$$
\mathbf{J}=\left[\begin{array}{cccccccc}
0 & 0 & 0 & 0 & -1 & 0 & 0 & 0 \\
0 & 0 & 0 & 0 & 0 & -1 & 0 & 0 \\
0 & 0 & 0 & 0 & 0 & 0 & -1 & 0 \\
0 & 0 & 0 & 0 & 0 & 0 & 0 & -1 \\
1 & 0 & 0 & 0 & 0 & 0 & 0 & 0 \\
0 & 1 & 0 & 0 & 0 & 0 & 0 & 0 \\
0 & 0 & 1 & 0 & 0 & 0 & 0 & 0 \\
0 & 0 & 0 & 1 & 0 & 0 & 0 & 0
\end{array}\right]
$$

The symbol $S(Z)$ is used for the Hamiltonian function because it can be related to the momentum flux associated with the Boussinesq model (5.14).

Written out the Hamiltonian system (5.18) is

$$
\begin{aligned}
-\frac{d \chi}{d x} & =\frac{\partial S}{\partial \gamma}=0 \\
-\frac{d q_{1}}{d x} & =\frac{\partial S}{\partial \phi_{1}}=0 \\
-\frac{d q_{2}}{d x} & =\frac{\partial S}{\partial \phi_{2}}=0 \\
-\frac{d H}{d x} & =\frac{\partial S}{\partial h_{1}}=\chi-\left(\rho_{1}-\rho_{2}\right) g h_{1}-\frac{1}{2} \frac{q_{1}^{2}}{\rho_{1} h_{1}^{2}}+\frac{1}{2} \frac{q_{1}^{2}}{\rho_{1}\left(d-h_{1}\right)^{2}} \\
\frac{d \gamma}{d x} & =\frac{\partial S}{\partial r}=h_{1} \\
\frac{d \phi_{1}}{d x} & =\frac{\partial S}{\partial q_{1}}=\frac{q_{1}}{\rho_{1} h_{1}} \\
\frac{d \phi_{2}}{d x} & =\frac{\partial S}{\partial q_{2}}=\frac{q_{2}}{\rho_{2}\left(d-h_{1}\right)} \\
\frac{d h_{1}}{d x} & =\frac{\partial S}{\partial H}=\frac{1}{\tau} H .
\end{aligned}
$$

Using the definitions $u_{j}=\frac{d \phi_{j}}{d x}$ for $j=1,2$, the $2^{\text {nd }}, 3^{\text {rd }}, 6^{\text {th }}$ and $7^{\text {th }}$ equations combine to give the first two equations of the steady part of (5.14). The $5^{\text {th }}$ equation is just a definition for $\gamma$. The $1^{\text {st }}, 4^{\text {th }}$ and $8^{\text {th }}$ equations combine to give

$$
0=\chi_{x}=\frac{d}{d x}\left(\left(\rho_{1}-\rho_{2}\right) g h_{1}+\frac{1}{2} \frac{q_{1}^{2}}{\rho_{1} h_{1}^{2}}-\frac{1}{2} \frac{q_{2}^{2}}{\rho_{1}\left(d-h_{1}\right)^{2}}\right)-\tau \frac{d^{3} h_{1}}{d x^{3}}
$$

which recovers the steady part of the third equation in (5.14).

\subsection{Properties of the bifurcating solitary waves}

Uniform flows correspond to a 3-parameter family of relative equilibria of this system. We will not go into the details of this construction as the system (5.18) is simple enough that the construction can be done explicitly, while still emphasizing the geometry of the bifurcation.

The uniform flow plus a perturbation takes the form

$$
\begin{aligned}
\gamma(x) & =h_{1} x+\widehat{\gamma}(x), & h_{1}(x) & =h_{1}+\widehat{h}(x), \quad H(x)=0+\widehat{H}(x) \\
\phi_{1}(x) & =u_{1} x+\widehat{\phi}_{1}(x), & \phi_{2}(x) & =u_{2} x+\widehat{\phi}_{2}(x) \\
\chi(x) & =\mathscr{R}+\widehat{\chi}(x), & \mathscr{R} & =\left(\rho_{1}-\rho_{2}\right) g h_{1}+\frac{1}{2} \rho_{1}\left(u_{1}\right)^{2}-\frac{1}{2} \rho_{2}\left(u_{2}\right)^{2} \\
q_{1}(x) & =\mathscr{Q}_{1}+\widehat{q}_{1}(x), & \mathscr{Q}_{1} & =\rho_{1} h_{1} u_{1} \\
q_{2}(x) & =\mathscr{Q}_{2}+\widehat{q}_{2}(x), & \mathscr{Q}_{2} & =\rho_{2}\left(d-h_{1}\right) u_{2},
\end{aligned}
$$

with $\left(h_{1}, u_{1}, u_{2}\right)$ near a critical uniform flow. Substitution into the governing equations leads to

$$
\begin{aligned}
-\frac{d \widehat{\chi}}{d x} & =0, \quad-\frac{d \widehat{q}_{1}}{d x}=0, \quad-\frac{d \widehat{q}_{2}}{d x}=0 \\
-\frac{d \widehat{H}}{d x} & =n_{1} \widehat{\chi}+n_{2} \widehat{q}_{1}+n_{3} \widehat{q}_{2}+\left(\rho_{1}-\rho_{2}\right) g\left(F_{1}^{2}+r F_{2}^{2}-1+\cdots\right) \widehat{h}-\frac{1}{2} \kappa \widehat{h}^{2}+\cdots \\
\frac{d \widehat{\gamma}}{d x} & =\widehat{h} \\
\frac{d \phi_{1}}{d x} & =\frac{1}{\rho_{1} h_{1}} \widehat{q}_{1}-\frac{u_{1}}{h_{1}} \widehat{h}+\cdots \\
\frac{d \phi_{2}}{d x} & =\frac{1}{\rho_{2}\left(d-h_{1}\right)} \widehat{q}_{2}+\frac{u_{2}}{\left(d-h_{1}\right)} \widehat{h}+\cdots \\
\frac{d \widehat{h}}{d x} & =\frac{1}{\tau} \widehat{H} .
\end{aligned}
$$


where $\mathbf{n}=\left(n_{1}, n_{2}, n_{3}\right)$ is in the kernel of $\mathrm{DP}(\mathbf{c})$ (cf. equation (4.9)) and

$$
\kappa=3\left(\rho_{1} \frac{u_{1}^{2}}{h_{1}^{2}}-\rho_{2} \frac{u_{2}^{2}}{\left(d-h_{1}\right)^{2}}\right) .
$$

Remarkably, the coefficient $\kappa$ of the nonlinear term is predicted by the geometry of the criticality surface discussed in $\S 4$. The value of this coefficient agrees with results in the literature on weakly nonlinear solitary waves $[19,4,11,12,13,15,18,20]$. What is new here is the connection with the geometry of criticality surfaces.

The $5^{\text {th }}, 6^{\text {th }}$ and $7^{\text {th }}$ equations of (5.20) just give drift along the $\widehat{\gamma}, \widehat{\phi}_{1}$ and $\widehat{\phi}_{2}$ directions, and the $1^{\text {st }}, 2^{\text {nd }}$ and $3^{\text {rd }}$ equations correspond to conservation of (Bernoulli) energy and mass flux. The important equations for solitary waves are the $4^{\text {th }}$ and $8^{\text {th }}$ equations which combine to give

$$
-\tau \widehat{h}_{x x}=I-\frac{1}{2} \kappa \widehat{h}^{2}+\cdots .
$$

The term proportional to $\widehat{h}$ drops out to leading order when the uniform flow is taken to be critical. The coefficient $I$ is defined by

$$
I=n_{1} \widehat{r}+n_{2} \widehat{q}_{1}+n_{3} \widehat{q}_{2}=n_{1}(R-\mathscr{R})+n_{2}\left(Q_{1}-\mathscr{Q}_{1}\right)+n_{3}\left(Q_{2}-\mathscr{Q}_{2}\right),
$$

where $\left(\mathscr{R}, \mathscr{Q}_{1}, \mathscr{Q}_{2}\right)$ is any point on the criticality surface away from the singularity $\kappa=0$. The coefficient $I$ gives the normal distance from the criticality surface in $\left(R, Q_{1}, Q_{2}\right)$ space.

Neglecting the higher order terms in (5.22), there is an explicit sech ${ }^{2}$ solitary wave solution. $\operatorname{sign}(\kappa)=\operatorname{sign}(I)$ is a necessary condition for the existence of solitary waves, and the sign of $I$ determines which side of the criticality surface the solitary waves exist. Once the sign of the chosen normal vector $\mathbf{n}$ is fixed, the sign of $I$ determines whether the solitary waves exist in the $+\mathbf{n}(I>0)$ or $-\mathbf{n}(I<0)$ direction. The sign of $\tau$ then determines whether the solitary waves are elevation or depression: if $\operatorname{sign}(\tau)=\operatorname{sign}(\kappa)$ the solitary wave is one of depression and if $\operatorname{sign}(\tau)=-\operatorname{sign}(\kappa)$ the solitary wave is one of elevation. An explicit expression for the solitary wave in the truncated normal form is

$$
\widehat{h}(x)=\nu\left(1-3 \operatorname{sech}^{2}(\gamma x)\right),
$$

with

$$
\nu=\frac{|\tau|}{\kappa \tau} \sqrt{2 \kappa I} \text { and } \quad \gamma=\left(\frac{\kappa I}{8 \tau^{2}}\right)^{1 / 4}
$$

In this normal form the conjugate flows (shown graphically in Figure 10) appear as $\pm(2 I / \kappa)^{1 / 2}$.

\section{Concluding Remarks}

A similar analysis can be done for two-layer flow with a free surface. The surface of criticality has higher dimension since $h_{1}$ and $h_{2}$ are independent coordinates, and $R_{1}$ and $R_{2}$ are independent. The surface of criticality generalizing Figure 8 is a three-dimensional hypersurface in $\left(h_{1}, h_{2}, u_{1}, u_{2}\right)$ space.

One of the reasons that the geometric view of criticality proposed in this paper is of interest is that it generalizes to criticality of non-trivial steady states. In [9] the criticality problem for Stokes waves coupled to a mean flow (there called secondary criticality) is formulated. Secondary criticality then leads to a secondary bifurcation of steady dark solitary waves. Dark solitary waves generalize the bifurcation of classical solitary waves associated with critical uniform flows. They are waves which are asymptotic to periodic states as $x \rightarrow \pm \infty$ but have local tanh-like behaviour for $|x|$ small, and the interior region has smaller amplitude than the periodic state at large $|x|$. A typical example is shown in Figure 1 of [9]. The term "dark solitary wave" is derived from optics where the small amplitude in the localized interior region is indicative of a region of low intensity light. 
The theory of secondary criticality can be applied to two-layer flows. In addition to the functionals $\left(R, Q_{1}, Q_{2}\right)$ one includes $B$, the wave action flux, and in addition to $\left(h_{1}, u_{1}, u_{2}\right)$ one includes $k$ the wavenumber of the periodic wave at the interface. Then one can show that the coupled system is critical when the determinant of the extended Jacobian vanishes,

$$
\operatorname{det}\left[\frac{\partial\left(R, Q_{1}, Q_{2}, B\right)}{\partial\left(h_{1}, u_{1}, u_{2}, k\right)}\right]=0 .
$$

When this condition is satisfied for periodic waves at the interface between two fluids with a rigid lid coupled to a 3-component mean flow - and there is good reason to suspect that it is satisfied for some parameter values - application of the theory in [9] would lead to the secondary bifurcation of dark solitary waves at the interface.

- Appendix -

\section{A Including the lid pressure in calculation of uniform flows}

The lid pressure can be included in the calculation of uniform flows by treating it as a Lagrange multiplier associated with the depth constraint $d=h_{1}+h_{2}$. In this case the Bernoulli energy in each layer is retained. Given values of the Bernoulli energy in the two layers, $R_{1}$ and $R_{2}$, values of the mass flux in each layer, $Q_{1}$ and $Q_{2}$, and the value of the total depth $d$, uniform flows consist of values of $h_{1}, h_{2}, u_{1}, u_{2}$ and $p_{s}$ satisfying

$$
\begin{aligned}
R_{1} & =\frac{1}{2} \rho_{1} u_{1}^{2}+\rho_{1} g h_{1}+\rho_{2} g h_{2}+p_{s}:=\frac{\partial \mathscr{F}}{\partial h_{1}} \\
R_{2} & =\frac{1}{2} \rho_{2} u_{2}^{2}+\rho_{2} g h_{1}+\rho_{2} g h_{2}+p_{s}:=\frac{\partial \mathscr{F}}{\partial h_{2}} \\
Q_{1} & =\rho_{1} h_{1} u_{1}:=\frac{\partial \mathscr{F}}{\partial u_{1}} \\
Q_{2} & =\rho_{2} h_{2} u_{2}:=\frac{\partial \mathscr{F}}{\partial u_{2}} \\
d & =h_{1}+h_{2}:=\frac{\partial \mathscr{F}}{\partial p_{s}},
\end{aligned}
$$

where

$$
\mathscr{F}=\frac{1}{2} \rho_{1} h_{1} u_{1}^{2}+\frac{1}{2} \rho_{2} h_{2} u_{2}^{2}+\frac{1}{2} \rho_{1} g h_{1}^{2}+\rho_{2} g h_{1} h_{2}+\frac{1}{2} \rho_{2} g h_{2}^{2}+p_{s}\left(h_{1}+h_{2}\right) .
$$

The flow is critical when the Jacobian of this mapping is degenerate. The Jacobian is

$$
\frac{\partial\left(R_{1}, R_{2}, Q_{1}, Q_{2}, d\right)}{\partial\left(h_{1}, h_{2}, u_{1}, u_{2}, p_{s}\right)}=\left[\begin{array}{ccccc}
\rho_{1} g & \rho_{2} g & \rho_{1} u_{1} & 0 & 1 \\
\rho_{2} g & \rho_{2} g & 0 & \rho_{2} u_{2} & 1 \\
\rho_{1} u_{1} & 0 & \rho_{1} h_{1} & 0 & 0 \\
0 & \rho_{2} u_{2} & 0 & \rho_{2} h_{2} & 0 \\
1 & 1 & 0 & 0 & 0
\end{array}\right]
$$

and

$$
\operatorname{det}\left[\frac{\partial\left(R_{1}, R_{2}, Q_{1}, Q_{2}, d\right)}{\partial\left(h_{1}, h_{2}, u_{1}, u_{2}, p_{s}\right)}\right]=\rho_{1} \rho_{2}\left[\rho_{1} h_{2} u_{1}^{2}+\rho_{2} h_{1} u_{2}^{2}-\left(\rho_{1}-\rho_{2}\right) g h_{1} h_{2}\right],
$$

recovering the familiar condition for criticality. As expected, the criticality condition is independent of the value of $p_{s}$. An observation that arises from the formulation including $p_{s}$ is that

$$
p_{s}=\frac{\partial S^{p h y s}}{\partial d}, \quad \text { with } h_{1}, h_{2}, u_{1}, u_{2} \text { fixed }
$$

where $S^{\text {phys }}$ is the physical momentum flux. 


\section{B The momentum flux}

The reduced momentum flux used in $\S 3$ is the natural conserved quantity when one uses the reduced energy, $R=R_{1}-R_{2}$, which factors out the lid pressure $p_{s}$. It differs from the physical momentum flux $S^{\text {phys }}$ by a constant.

The physical momentum is $I=\rho_{1} h_{1} u_{1}+\rho_{2} h_{2} u_{2}$, and using the governing equations $(2.1)$ the conservation law for $I$ can be constructed

$$
\frac{\partial I}{\partial t}+\frac{\partial S^{p h y s}}{\partial x}=0
$$

with

$$
S^{\text {phys }}=\rho_{1} h_{1} u_{1}^{2}+\rho_{2} h_{2} u_{2}^{2}+\frac{1}{2} \rho_{1} g h_{1}^{2}+\rho_{2} g h_{1} h_{2}+\frac{1}{2} \rho_{2} g h_{2}^{2}+p_{s} d .
$$

Taking $h_{2}=d-h_{1}$ this simplifies to

$$
S^{\text {phys }}=\rho_{1} h_{1} u_{1}^{2}+\rho_{2}\left(d-h_{1}\right) u_{2}^{2}+\frac{1}{2}\left(\rho_{1}-\rho_{2}\right) g h_{1}^{2}+\frac{1}{2} \rho_{2} g d^{2}+p_{s} d .
$$

The difference between $S$ and $S^{p h y s}$ is

$$
S^{\text {phys }}=S+R_{2} d-\frac{1}{2} \rho_{2} g d^{2},
$$

where

$$
R_{2}=\frac{1}{2} \rho_{2} u_{2}^{2}+\rho_{2} g h_{1}+\rho_{2} g h_{2}+p_{s},
$$

is the flux of $\rho_{2} u_{2}$ (see equation (2.1)). Either $S$ or $S^{\text {phys }}$ can be used in the diagrams in $\S 3$. Since the values of $R_{2}$ and $d$ are chosen independently of $h_{1}, u_{1}$ and $u_{2}$, the only effect of changing $S$ to $S^{\text {phys }}$ is a shift of the diagrams up or down in the $S$ direction.

\section{Acknowledgements}

Helpful comments from Roger Grimshaw are gratefully acknowledged. The work of Neil Donaldson was supported by a PhD studentship awarded by EPSRC.

\section{References}

[1] L. Armi. The hydraulics of two flowing layers with different densities, J. Fluid Mech. 163 $27-58$ (1986).

[2] P.G. BAInes. Topographic effects in Stratified Flows, Cambridge University Press (1995).

[3] V.I. Arnold, S.M. Gusein-Zade \& A.N. Varchenko. Singularities of Differentiable Maps, Volume I, Birkhäuser: Boston (1985).

[4] T.B. Benjamin. Internal waves of finite amplitude and permanent form, J. Fluid Mech. 25 241-270 (1966).

[5] T.B. Benjamin \& T.J. Bridges. Reappraisal of the Kelvin-Helmholtz instability. Part 1. Hamiltonian structure, J. Fluid Mech. 333 301-325 (1997).

[6] T.B. Benjamin \& M.J. Lighthill. On cnoidal waves and bores, Proc. Royal Soc. London A 224 448-460 (1954).

[7] T.J. BRIDGes. Degenerate relative equilibria, curvature of the momentum map, and homoclinic bifurcation, Preprint, University of Surrey (2006). 
[8] T.J. Bridges \& N.M. Donaldson. Degenerate periodic orbits and homoclinic torus bifurcation, Phys. Rev. Lett. 95(10) 104301 (2005).

[9] T.J. Bridges \& N.M. Donaldson. Secondary criticality of water waves. Part 1. Definition, bifurcation and solitary waves, J. Fluid Mech. 565 381-417 (2006).

[10] J. BüHLER. Simple internal waves and bores, J. Hydr. Engrg. 120 638-645 (1994).

[11] W. Choi \& R. Camassa. Fully nonlinear internal waves in a two-fluid system, J. Fluid Mech. 396 1-36 (1999).

[12] W. Craig, P. Guyenne \& H. Kalisch. Hamiltonian long-wave expansions for free surfaces and interfaces, Comm. Pure Appl. Math. 58 1587-1641 (2005).

[13] F. Dias \& J.-M. Vandenbroeck. On internal fronts, J. Fluid Mech. 479 145-154 (2003).

[14] N.M. Donaldson. Criticality theory and conformal mapping techniques for single and two-layer water-wave systems, PhD Thesis, University of Surrey (2006).

[15] M. FunAKishi \& M. OiKawa. Long internal waves of large amplitude in a two-layer fluid, J. Phys. Soc. Japan 55 128-144 (1986).

[16] R.H.J. Grimshaw. Internal solitary waves, Adv. in Fluid Mech. V, 209-218, Adv. Fluid Mech. 40, WIT Press: Southampton (2004).

[17] K.R. Helfrich \& W.K. Melville. Long nonlinear internal waves, Ann. Rev. Fluid Mech. 38 395-425 (2006).

[18] O. LAGET \& F. Dias. Numerical computation of capillary-gravity interfacial solitary waves, J. Fluid Mech. 349 221-251 (1997).

[19] R.R. Long. Solitary waves in the one and two-fluid system, Tellus 8 460-471 (1956).

[20] D.I. Pullin \& R.H.J. GRimshaw. Finite-amplitude solitary waves at the interface between two homogeneous fluids, Phys. Fluids 31 3550-3559 (1988).

[21] M.J. Sewell. Maximum and Minimum Principles, Cambridge University Press (1987).

[22] M.J. Sewell \& D. Porter. Constitutive surfaces in fluid mechanics, Math. Proc. Camb. Phil. Soc. 88 517-546 (1980). 